\title{
Executive functioning affects health behaviour in older people too
}

\author{
David McMinn research fellow ${ }^{1}$, Julia Allan lecturer ${ }^{2}$, Michael Daly senior lecturer ${ }^{3}$ \\ ${ }^{1}$ Rowett Institute of Nutrition and Health, School of Medicine and Dentistry, Aberdeen AB25 2ZD, UK; ${ }^{2}$ Health Psychology Group, School of Medicine \\ and Dentistry, Aberdeen, UK; ${ }^{3}$ Behavioural Science Centre, Stirling Management School, University of Stirling, Stirling, UK
}

Marteau and Hall provide interesting insight into the important links between early life environments, poverty, deleterious health behaviours, and the cognitive abilities used to control such behaviours-the "executive functions."1 They suggest that associations between early life exposure to poverty and subsequent reductions in executive functions contribute to health inequalities. They also acknowledge the possibility of bidirectionality in this association: "those starting life with the strongest executive functioning are those who are more likely to engage in the behaviours that most nurture this brain system as well as longer term health."

Given our ageing population and the requirement to reduce age related cognitive disease, we also a need to improve our understanding of the associations between health behaviours and executive functions in older people, while acknowledging the potential for bidirectionality. We investigated the association between executive functions and physical activity in a large longitudinal sample of older people from the English Longitudinal Study of Ageing. ${ }^{2}$ We hypothesised that physical activity would improve executive functioning through fitness related reductions in brain atrophy, ${ }^{3}$ but that the efficiency of people's executive functions might also play an important role in initiating future engagement in physical activity. ${ }^{4}$

We identified a reciprocal mutually enhancing association - those with physically active lifestyles showed subsequent increases in executive function and, crucially, those with poor executive function tended to show large decreases in rates of participation in physical activity over time. ${ }^{5}$ Our findings are encouraging for behaviour change practitioners because they identify two potential mechanisms for improving health. Interventions could be aimed at executive functions and physical activity concurrently to provide a more potent and cumulative approach to improving health.

We support Marteau and Hall's notion that executive functioning and health related behaviour are linked by positive and negative feedback loops. A better understanding of these reciprocal associations is vital to pinpoint phases amenable to intervention in this feedback loop.

\section{Competing interests: None declared.}

1 Marteau TM, Hall PA. Breadlines, brains, and behaviour. BMJ 2013;347:f6750. (12 November.)

2 Marmot M, Banks JA, Blundell R, Lessof C, Nazroo J. Health, wealth and lifestyles of the older population in England: the 2002 English Longitudinal Study of Ageing. Institute for Fiscal Studies, 2003. www. ifs.org.uk/publications/3088.

3 Weinstein AM, Voss MW, Prakash RS, Chaddock L, Szabo A, White SM, et al. The association between aerobic fitness and executive function is mediated by prefrontal cortex volume. Brain Behav Immun 2012;26:811-9.

4 Hall PA, Fong GT, Epp LP, Elias, LJ. Executive function moderates the intention-behaviour link for physical activity and dietary behavior. Psych Health 2008;23:309-26.

5 Daly M, McMinn D, Allan JL. A bidirectional relationship between physical activity and executive function in older adults. Working paper. University of Aberdeen. http://hdl.handle. net/2164/3012. 